\title{
A realistic approach to inclusive $e$-scattering from nuclei
}

\author{
R. Schiavilla \\ Department of Physics, Old Dominion University, Norfolk, VA 23529 \\ Theory Center, Jefferson Lab, Newport News, VA 23606
}

\begin{abstract}
We review the current status of calculations, based on realistic nuclear interactions and currens, of the inclusive electromagnetic response of nuclei in the quasi-elastic region.
\end{abstract}

Keywords: inclusive electron scattering from nuclei

PACS: $24.10 . \mathrm{Cn}, 25.30 .-\mathrm{c}$

\section{INTRODUCTION}

In this talk, we review our current understanding of the inclusive electromagnetic response of nuclei in the quasielastic region within a dynamical approach based on realistic nuclear interactions and currents. These interactions and currents are discussed in the next section. The calculation of inclusive electromagnetic (or weak) response functions is a challanging theoretical problem. In light nuclei, two different approaches have been pursued so far, one based on sum rules and the other utilizing integral-transform techniques aimed at removing the need of calculating explicitly the nuclear excitation spectrum. These approaches are reviewed in the section after next. The prospects for extending these methods to a calculation of neutrino inclusive response induced by charge-changing and neutral weak currents are briefly outlined in the last section.

\section{REALISTIC NUCLEAR INTERACTIONS AND ELECTROMAGNETIC CURRENTS}

The two-nucleon $(N N)$ potential consist of a long-range component induced by one-pion exchange (OPE) and intermediate- to short-range components which are modeled phenomenologically, as in the Argonne $v_{18}$ (AV18) potential [1], or by scalar and vector meson-exchanges, as in the CD-Bonn potential [2], or by a combination of twopion-exchange mechanisms and contact two-nucleon terms, such as in the chiral-effective-field-theory potentials [3]. All these potential models fit the $N N$ database for energies up to the pion production threshold with $\chi^{2} \simeq 1$. However, it is by now well established that $N N$ potentials alone fail to predict the spectra of light nuclei [4], cross sections and analyzing powers in $N d$ scattering at low [5] and intermediate [6] energies, and the nuclear matter equilibrium properties [7].

Models of the three-nucleon $(N N N)$ potential include two- and three-pion exchange [8,9] as well as short-range repulsive terms. In the Illinois model 7 (IL7), these multi-pion exchange components involve excitation of intermediate $\Delta$ resonances. The IL7 strength is determined by four parameters which are fixed by a best fit to the energies of about 17 low-lying states of nuclei in the mass range $A \leq 10$, obtained in combination with the AV18 NN potential. The AV18/IL7 Hamiltonian reproduces well the spectra of nuclei with $A=9-12$ [10]—in particular, the attraction provided by the IL7 $N N N$ potential in isospin 3/2 triplets is crucial for the $p$-shell nuclei-and the $p$-wave resonances with $J^{\pi}=(3 / 2)^{-}$and $(1 / 2)^{-}$in $n^{-4} \mathrm{He}$ scattering [11].

Realistic models for the electromagnetic charge and current operators include one- and two-body parts. The onebody charge and current operators follow from a non-relativistic expansion of the single-nucleon four-current. The two-body current operators are separated into model-independent (MI) and model-dependent (MD) terms [12]. The MI terms are derived from the $N N$ potential (the AV18 in the present case), and their longitudinal components satisfy, by construction, current conservation with it. They contain no free parameters, and their short-range behavior is consistent with that of the potential. The dominant terms, isovector in character, originate from the static part of the potential, which is assumed to be due to exchanges of effective pseudoscalar ( $\pi$-like) and vector ( $\rho$-like) mesons. At large internucleon separations, where the $N N$ potential is driven by the OPE mechanism, the MI currents coincide with the well known seagull and in-flight OPE currents. 
The MD currents are purely transverse, and unconstrained by current conservation. The dominant term is associated with excitation of intermediate $\Delta$ isobars [13]. Additional (and numerically small) MD currents arise from the isoscalar $\rho \pi \gamma$ and isovector $\omega \pi \gamma$ transition mechanisms. These MI and MD currents have been shown to reproduce satisfactorily a variety of nuclear properties and reactions, including magnetic moments of, and $M 1$ transitions between, lowlying states of light nuclei [14], the trinucleon magnetic form factors [12], and radiative captures in the few-nucleon systems [15].

The two-body charge operators represent relativistic corrections: they vanish at zero momentum transfer because of charge conservation; they also vanish in the static limit. The leading term is due to OPE, and is derived from an analysis of the virtual pion photo-production amplitude. Additional (and numerically small) contributions arise from exchange of vector mesons and $\rho \pi \gamma$ and $\omega \pi \gamma$ mechanisms. These two-body charge operators, in particular the OPE one, are essential for reproducing the observed charge form factors of the hydrogen and helium isotopes (see Ref. [16] and references therein).

\section{APPROACHES TO INCLUSIVE SCATTERING}

Two response functions characterize inclusive $\left(e, e^{\prime}\right)$ scattering, defined as

$$
R_{\alpha}(q, \omega)=\sum_{f \neq 0} \delta\left(\omega+E_{0}-E_{f}\right)\left|\left\langle f\left|O_{\alpha}(\mathbf{q})\right| 0\right\rangle\right|^{2},
$$

where $|0\rangle$ and $|f\rangle$ denote, respectively, the initial and final nuclear states of energies $E_{0}$ and $E_{f}, \omega$ and $\mathbf{q}$ are the electron energy and momentum transfers, and $O_{\alpha}(\mathbf{q})$ is either the nuclear charge $(\alpha=L)$ or current $(\alpha=T)$ operator. Major complications in their calculation arise in consequence of the need of, and technical difficulties associated with, providing an accurate description of the initial bound- and final scattering-state wave functions, based on realistic interactions. These complications can be avoided, at least in part, either by studying integral properties of the response functions-i.e., longitudinal and transverse sum rules—or by using integral transform techniques of the type

$$
E(q, \tau)=\int_{0}^{\infty} \mathrm{d} \omega K(\tau, \omega) R(q, \omega),
$$

which for a suitable choice of kernel—for example, Laplace [17] or Lorentz (see Ref. [18] and references therein) allows the use of closure over $|f\rangle$, thus removing the need of explicitly calculating the nuclear excitation spectrum. While in principle exact, both these approaches have drawbacks.

\section{Sum rules}

Longitudinal (Coulomb) and transverse sum rules can be expressed as ground-state expectation values of the charge and current operators:

$$
S_{\alpha}(q)=C_{\alpha} \int_{\omega_{\mathrm{th}}^{+}}^{\infty} \mathrm{d} \omega \frac{R_{\alpha}(q, \omega)}{G_{E p}^{2}(q, \omega)}=C_{\alpha}\left[\left\langle 0\left|O_{\alpha}^{\dagger}(\mathbf{q}) O_{\alpha}(\mathbf{q})\right| 0\right\rangle-\left|\left\langle 0\left|O_{\alpha}(\mathbf{q})\right| 0\right\rangle\right|^{2}\right],
$$

where it is understood that the charge and current operators $O_{\alpha}(\mathbf{q})$ have been divided by the proton electric form factor $G_{E p}$, and the $C_{\alpha}$ are normalization constants such that, in the limit $q \rightarrow \infty$ and under the approximation that only one-body terms are retained in $O_{\alpha}(\mathbf{q})$, then $S_{\alpha}(q \rightarrow \infty)=1$ [19].

The longitunal and transverse sum rules defined above (as well as energy-weighted ones) have been calculated exactly with quantum Monte Carlo techniques in $A=2-6$ nuclei [19, 20, 21]. However, direct comparison between these and the experimentally extracted sum rules cannot be made unambiguously for two reasons. First, the experimental determination of the sum rules requires measuring the associated response functions over the whole energy transfer, from threshold to $\infty$. Inclusive electron scattering only allows access to the space-like region of the four momentum transfer $\omega<q$. Therefore, for a meaningful comparison between theory and experiment one needs to estimate the strength outside the region covered by experiment, either by extrapolating the data or by parametrizing the high energy tail and using energy-weighted sum rules to constrain it.

The second reason that makes the direct comparison between theoretical and "experimental" sum rules difficult lies in the inherent inadequacy of the present theoretical model for the nuclear electromagnetic current, in particular, its 
lack of explicit pion production mechanisms. The latter mostly affect the transverse response and make the $\Delta$ peak outside the boundary of applicability of the present theory. However, the charge and current operator discussed in the previous section provide a realistic and quantitative description of the longitudinal and transverse response function in the quasi-elastic peak region, where nucleon and virtual pion degrees of freedom are dominant.

Experimental Coulomb sum rules in the few-nucleon systems are in good agreement with data (after inclusion of tail contributions), as shown in Fig. 1 [21]. Contributions from two-body terms and one-body relativistic corrections in the charge operator play a minor role in the momentum transfer range covered by experiment. However, the situation in reference to the Coulomb sum of medium- and heavy-weight nuclei is still controversial, that is, the question of whether the longitudinal response in these systems is quenched or not is yet to be resolved satisfactorily.

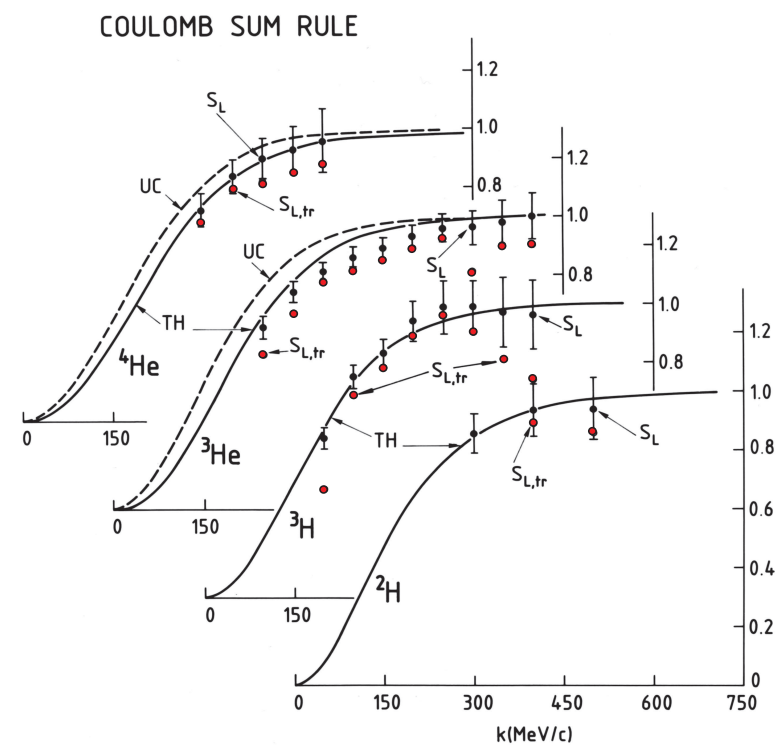

FIGURE 1. The longitudinal (Coulomb) sum rule in the $A=2-4$ nuclei: theory (solid lines) versus experiment (solid circles with error bars); circles without error bars do not include tail corrections.

The ratios of transverse to longitudinal sum rules in the $A=3-6$ nuclei are shown in Fig. 2. The transverse sum rule is substantially increased by two-body current contributions. One interesting feature of the resulting enhancement is that it increases, for fixed $q$, in going from $A=3$ to 4 , and decreases from $A=4$ to 6 . It has been shown in Ref. [19] that the excess transverse strength, defined as $\Delta S_{T}(q)=S_{T}(q)-S_{T}(q ; 1 \mathrm{~b})$ and where the label "1b" means one-body, is proportional to
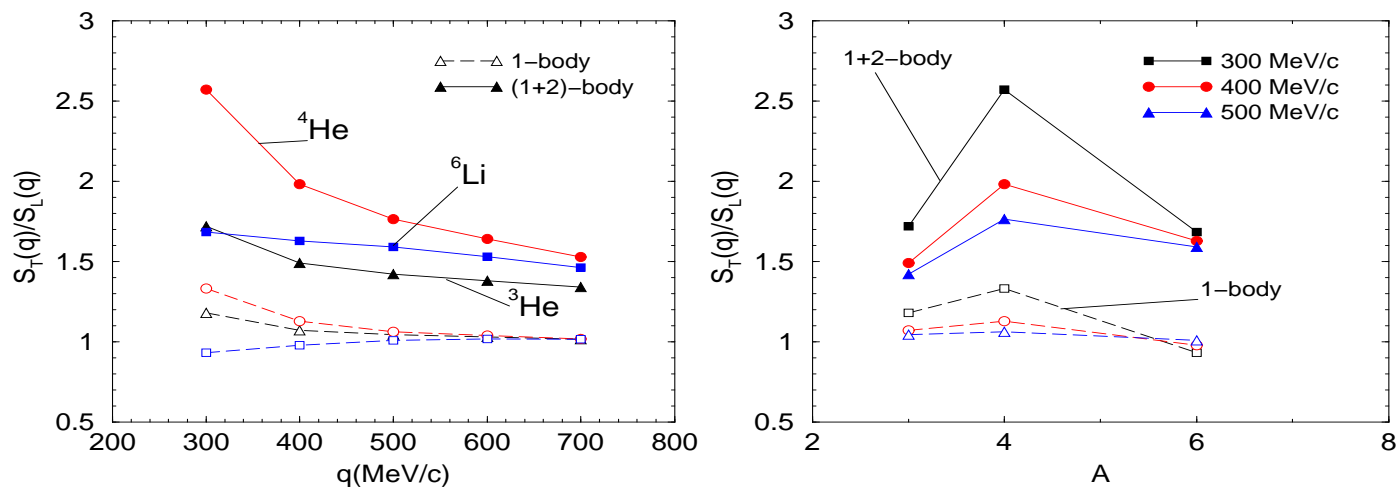

FIGURE 2. The ratio of transverse to longitudinal sum rules, obtained with one-body only and one- plus two-body terms in the charge and current operators, as function of the momentum transfer in ${ }^{3} \mathrm{He},{ }^{4} \mathrm{He}$, and ${ }^{6} \mathrm{Li}$ (left panel) or as function of mass number for $q=300,400$, and $500 \mathrm{MeV} / \mathrm{c}$ (right panel).

$$
\Delta S_{T}^{A}(q) \simeq C_{T} \int_{0}^{\infty} \mathrm{d} x \operatorname{tr}\left[F(x ; q) \rho^{A}(x ; p n)\right]_{\sigma \tau} .
$$


Here $F$ is a complicated matrix in the two-nucleon spin-isospin space depending on the current operators, and $\rho^{A}$ is the $p n$ density matrix in this space as function of the relative distance, a quantity strongly affected by central and tensor correlations induced by the repulsive core at short range and OPE component at long range of the $N N$ potential. Thus the transverse enhancement is primarily due to $p n$ pairs, which can be in isospin $T=0$ and $T=1$. It is known [22] that these densities scale as

$$
\rho^{A}(x ; p n, T=0) \simeq R_{A} \rho^{d}(x)
$$

and similarly for $T=1 p n$ pairs with $\rho^{d} \rightarrow \rho^{q b}$, where the label $d$ and $q b$ denote, respectively, the deuteron and ${ }^{1} \mathrm{~S}_{0}$ quasi-bound states. The scaling factors for $T=0$ and $1 \mathrm{pn}$ pairs have been calculated in light nuclei, and have been found to be close to each other, with $R_{A}=2.0,4.7$, and 6.3 in ${ }^{3} \mathrm{He},{ }^{4} \mathrm{He}$, and ${ }^{6} \mathrm{Li}$, respectively. The calculated excess transverse strength is consistent with that expected on the basis of the scaling behavior above [19]. Furthermore, the analysis above suggests that two-body currents may enhance significantly the transverse response function in the quasi-elastic region.

\section{Euclidean response functions}

The Euclidean response functions are defined as

$$
\widetilde{E}_{\alpha}(q, \tau)=\int_{\omega_{\mathrm{th}}^{+}}^{\infty} \mathrm{d} \omega \mathrm{e}^{-\tau\left(\omega-E_{0}\right)} \frac{R_{\alpha}(q, \omega)}{G_{E p}^{2}(q, \omega)}=\left\langle 0\left|O_{\alpha}^{\dagger}(\mathbf{q}) \mathrm{e}^{-\tau\left(H-E_{0}\right)} O_{\alpha}(\mathbf{q})\right| 0\right\rangle-(\text { elastic term })
$$

and represent weighted sums of $R_{\alpha}(q, \omega)$. At $\tau=0$ they correspond to the sum rules discussed in the previous section, while their derivatives evaluated at $\tau=0$ correspond to energy-weighted sum rules. Below we present results for the scaled Euclidean responses $E(q, \tau)=\exp \left[q^{2} \tau /(2 m)\right] \widetilde{E}(q, \tau)$-inclusion of this factor removes the trivial energy dependence obtained from scattering off an isolated (non-relativistic) nucleon. The longitudinal and transverse Euclidean responses are, respectively, $Z$ and $Z \mu_{p}^{2}+(A-Z) \mu_{n}^{2}$ for a collection of $A$ non-interacting nucleons, $Z$ of which are protons ( $\mu_{p}$ and $\mu_{n}$ are the proton and neutron magnetic moments).

The main advantage of formulating the Euclidean response is that it can be calculated exactly using Green's function or path-integral Monte Carlo techniques, including both final state interactions and two-body components in the nuclear charge and current operators [17]. The calculated longitudinal and transverse Euclidean response functions
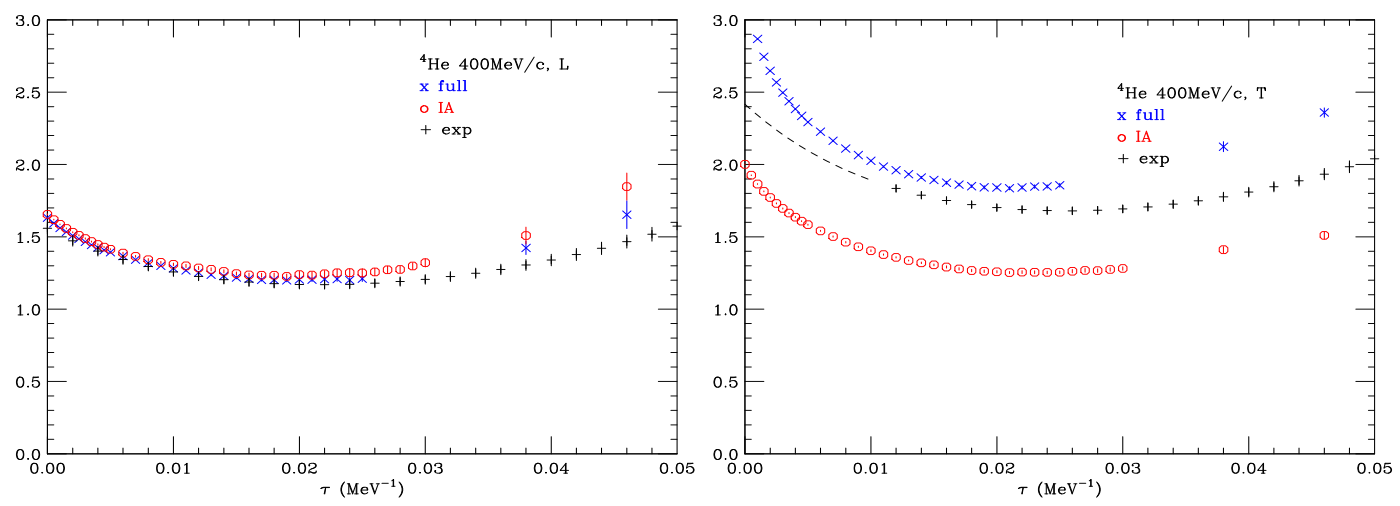

FIGURE 3. The Euclidean longitudinal and transverse response functions in ${ }^{4} \mathrm{He}$, obtained with one-body only (curve labelled IA) and one- plus two-body (curve labelled Full) terms in the charge and current operators, are compared to experiment.

of ${ }^{4} \mathrm{He}$ are displayed in Fig. 3 [19]. The experimental ones are obtained by Laplace-transforming the data, since a direct numerical inversion of $\widetilde{E}(q, \tau)$ is not possible due to the ill-posed nature of such a problem. In order not to include too much of the tail of the $\Delta$ resonance, the integration has been carried out up to the energy loss $\omega$ where the transverse response starts to increase significantly with $\omega$. Since for the transverse Euclidean response at very small $\tau$ the tail of the $\Delta$ peak nevertheless plays a role, the experimental response in this region is indicated by a dashed line only, and should not be compared to theoretical calculations. However, as $\tau$ increases beyond $\tau \simeq 0.01 \mathrm{MeV}^{-1}$, the Euclidean response probes strength in the quasi-elastic peak region. At $\tau>0.03 \mathrm{MeV}^{-1}$, contributions to $E(q, \tau)$ from this region is strongly suppressed, and the Euclidean response is mostly sensitive to strength at threshlold, which 
is poorly measured. The large enhancement of the transverse response between $(0.01 \leq \tau \leq 0.03) \mathrm{MeV}^{-1}$ due to two-body terms in the current operator should be noted.

\section{OUTLOOK}

It should be possible to use quantum Monte Carlo methods to study neutrino response functions, and associated sum rules, in light nuclei (including ${ }^{12} \mathrm{C}$ ) within the same realistic dynamical framework illustrated in this talk. In recent years, this has become a hot topic in view of the anomaly observed in recent neutrino quasi-elastic scattering data on ${ }^{12} \mathrm{C}$ [23], i.e., the excess, at relatively low energy, of measured cross section relative to theoretical calculations. Analyses based on these calculations have led to speculations that our present understanding of the nuclear response to charge-changing weak probes may be incomplete [24] and, in particular, that the momentum transfer dependence of the nucleon axial form factor [25] may be quite different from that inferred from analyses of pion electro-production data [26] and measurements of neutrino and anti-neutrino cross sections from proton and deuteron [27] However, the calculations on which these analyses are based use rather crude models of nuclear structure-Fermi gas or local-density approximations of nuclear matter spectral functions-as well as simplistic treatments of the reaction mechanism, and should therefore be viewed with skepticism.

As shown in this talk, "exact" calculations of the electromagnetic response functions in the $A=3-4$ nuclei [19] are in satisfactory agreement with data. In particular, these calculations have shown that the transverse response is significantly increased over the quasi-elastic peak region by two-body currents, in particular those associated with OPE. It will be interesting to see whether this mechanism is effective in the weak sector probed by neutrino scattering, and possibly provide an explanation for the observed anomaly in the $12 \mathrm{C}$ data. Work along these lines is in progress.

\section{ACKNOWLEDGMENTS}

I would like thank my collaborators J. Carlson, S. Gandolfi, L.E. Marcucci, S.C. Pieper, G. Shen, and R.B. Wiringa for

their many contributions to the research reported in this talk. I also would like to gratefully acknowledge the support of the U.S. Department of Energy, Office of Nuclear Science, under contract DE-AC05-06OR23177.

\section{REFERENCES}

1. R.B. Wiringa, V.G.J. Stoks, and R. Schiavilla, Phys. Rev. C 51, 38 (1995).

2. R. Machleidt, Phys. Rev. C 63, 024001 (2001).

3. D.R. Entem and R. Machledt, Phys. Rev. C 68, 041001 (2003); R. Machleidt and D.R. Entem, Phys. Rep. 503, 1 (2011).

4. S.C. Pieper and R.B. Wiringa, Ann. Rev. Nucl. Part. Sci. 51, 53 (2001).

5. L.E. Marcucci, A. Kievsky, L. Girlanda, S. Rosati, and M. Viviani, Phys. Rev. C 80, 034003 (2009).

6. N. Kalantar-Nayestanaki, private communication; St. Kystrin et al., Phys. Rev. C 72, 044006 (2005).

7. A. Akmal, V.R. Pandharipande, and D.G. Ravenhall, Phys. Rev. C 58, 1804 (1998).

8. S.C. Pieper, AIP Conf. Proc. 1011, 143 (2008).

9. H. Krebs, A. Gasparyan, and E. Epelbaum, Phys. Rev. C 85, 054006 (2012).

10. S.C. Pieper and R.B. Wiringa, private communication.

11. K.M. Nollett, S.C. Pieper, R.B. Wiringa, J. Carlson, and G.M. Hale, Phys. Rev. Lett. 99, 022502 (2007).

12. L.E. Marcucci, M. Viviani, R. Schiavilla, A. Kievsky, and S. Rosati, Phys. Rev. C 72, 014001 (2005).

13. R. Schiavilla, R.B. Wiringa, V.R. Pandharipande, and J. Carlson, Phys. Rev. C 45, 2628 (1992).

14. S. Pastore, S.C. Pieper, R. Schiavilla, and R.B. Wiringa, Phys. Rev. C in press.

15. L. Girlanda et al., Phys. Rev. Lett. 105, 232502 (2010).

16. J. Carlson and R. Schiavilla, Rev. Mod. Phys. 70, 743 (1998).

17. J. Carlson and R. Schiavilla, Phys. Rev. Lett. 68, 3682 (1992); Phys. Rev. C 49, R2880 (1994).

18. W. Leidemann and G. Orlandini, Prog. Part. and Nucl. Phys. 68, 158 (2013).

19. J. Carlson, J. Jourdan, R. Schiavilla, and I. Sick, Phys. Rev. C 65, 024002 (2002).

20. R. Schiavilla, Nucl. Phys. A499, 301 (1989).

21. R. Schiavilla, A. Fabrocini, and V.R. Pandharipande, Phys. Rev. C 40, 1484 (1989).

22. J.L. Forest et al., Phys. Rev. C 54, 646 (1996).

23. A.A. Aguilar-Arevalo et al., Phys. Rev. Lett. 100, 032301 (2008).

24. O. Benhar, P. Coletti, and D. Meloni, Phys. Rev. Lett. 105, 132301 (2010).

25. C. Juszczak, J.T. Sobczyk, and J. Zmuda, Phys. Rev. C 82, 045502 (2010). 
26. E. Amaldi, S. Fubini, and G. Furlan, Electroproduction at Low Energy and Hadron Form Factors (Springer Tracts in Modern Physics No. 83, 1979), p.1.

27. N.J. Baker et al., Phys. Rev. D 23, 2499 (1981); K.L. Miller et al., Phys. Rev. D 26, 537 (1982); T. Kitagaki et al., Phys. Rev. D 28, 436 (1983); L.A. Ahrens et al., Phys. Rev. D 35, 785 (1987). 